\title{
A GENERAL CLASS OF IMPULSIVE EVOLUTION EQUATIONS
}

\author{
JINRONG WANG — MiCHAL FEČKAN
}

\begin{abstract}
One of the novelty of this paper is the study of a general class of impulsive differential equations, which is more reasonable to show dynamics of evolution processes in Pharmacotherapy. This fact reduces many difficulties in applying analysis methods and techniques in Bielecki's normed Banach spaces and thus makes the study of existence and uniqueness theorems interesting. The other novelties of this paper are new concepts of Ulam's type stability and Ulam-Hyers-Rassias stability results on compact and unbounded intervals.
\end{abstract}

\section{Introduction}

The dynamic of evolution processes in the real world is often subjected to abrupt changes such as shocks, harvesting, and natural disasters. In general, these short-term perturbations are usually regarded as having acted instantaneously or appearing in the form of instantaneous impulses involving the corresponding differential equations. Many authors were devoted to study mild solutions to impulsive evolution equations with instantaneous impulses of the

2010 Mathematics Subject Classification. Primary: 35F55, 35R12; Secondary: 34G20, 37K45, 47D06.

Key words and phrases. Impulsive evolution equations, mild solutions, existence, stability.

The first author acknowledges the support by National Natural Science Foundation of China (11201091), Outstanding Scientific and Technological Innovation Talent Award of Education Department of Guizhou Province ([2014]240) and Doctor Project of Guizhou Normal College (13BS010). The second author acknowledges the support by Grants VEGA-MS 1/0071/14 and VEGA-SAV 2/0029/13 and by Slovak Research and Development Agency under the contract No. APVV-14-0378. 
form

$$
\begin{cases}x^{\prime}(t)=A x(t)+f(t, x(t)), & t \in J^{\prime}:=J \backslash\left\{t_{1}, \ldots, t_{m}\right\}, J:=[0, T], \\ x\left(t_{k}^{+}\right)=x\left(t_{k}^{-}\right)+I_{k}\left(x\left(t_{k}^{-}\right)\right), & k=1, \ldots, m,\end{cases}
$$

where the linear unbounded operator $A: D(A) \subseteq X \rightarrow X$ is the generator of a $C_{0}$-semigroup (analytic or compact) $\{T(t), t \geq 0\}$ on a Banach space $X$ with a norm $\|\cdot\|, f: J \times X \rightarrow X$ and $I_{k}: X \rightarrow X$ and fixed impulsive time $t_{k}$ satisfy $0=$ $t_{0}<t_{1}<\ldots, t_{m}<t_{m+1}=T$, the symbols $x\left(t_{k}^{+}\right):=\lim _{\varepsilon \rightarrow 0^{+}} x\left(t_{k}+\varepsilon\right)$ and $x\left(t_{k}^{-}\right):=$ $\lim _{\varepsilon \rightarrow 0^{-}} x\left(t_{k}+\varepsilon\right)$ represent the right and left limits of $x(t)$ at $t=t_{k}$, respectively. Note that $I_{k}$ in (1.1) is a sequences of instantaneously impulse operators and has been developed in physics, population dynamics, biotechnology, and so forth. For more details on differential equations with instantaneous impulses, one can see for instance the monographs [8], [10], [33], the works on not time variable impulses problem [4], [5], [12], [14]-[16], [26], [34], [36], [37] and time variable impulses problem [1]-[3], [17]-[19] and the references therein.

However, the action of instantaneous impulses seems do not describe some certain dynamics of evolution processes in Pharmacotherapy. Taking into consideration the hemodynamic equilibrium of a person, the introduction of the drugs in the bloodstream and the consequent absorption for the body are gradual and continuous process. Thus, we do not expect to use the model (1.1) to describe such process. In fact, the above situation is fallen in a new case of impulsive action, which starts at an arbitrary fixed point and stays active on a finite time interval. To this end, Hernández and O'Regan [21] introduce a new class of impulsive evolution equations (with not instantaneous impulses) of the form

$$
\begin{cases}x^{\prime}(t)=A x(t)+f(t, x(t)), & t \in\left(s_{i}, t_{i+1}\right], i=0, \ldots, m \\ x(t)=g_{i}(t, x(t)), & t \in\left(t_{i}, s_{i}\right], i=1, \ldots, m \\ x(0)=x_{0} \in X, & \end{cases}
$$

where $A$ and $f$ are the same as (1.1) and the fixed points $s_{i}$ and $t_{i}$ satisfy $0=t_{0}=s_{0}<t_{1}<s_{1}<t_{2}<\ldots<s_{m-1}<t_{m}<s_{m}<t_{m+1}=T$, and $g_{i}:\left[t_{i}, s_{i}\right] \times X \rightarrow X$ is continuous for all $i=1, \ldots, m$. Here $g_{i}$ is regarded as continuous action process.

The concepts of mild solutions and classical solutions are introduced by Hernández and O'Regan [21] (see Definitions 2.1 and 2.2). Meanwhile, existence and uniqueness results of (1.2) are presented by using the theory of strongly continuous semigroup and compact semigroup via fixed point theorems (see Theorems 2.1 and 2.2, [21] and Theorems 2.1 and 2.2, [29]). Next, Pierri et al. [29] continue the work and development in [21] and study the existence and uniqueness of mild solutions to semilinear impulsive differential equations with not 